\title{
Exploiting data in smart factories: real-time state estimation and model improvement in metal forming mass production
}

\author{
Jos Havinga ${ }^{1}$ (D) . Pranab K. Mandal ${ }^{2}$. Ton van den Boogaard ${ }^{1}$
}

Received: 5 March 2019 / Accepted: 16 June 2019 / Published online: 24 July 2019

(C) The Author(s) 2019

\begin{abstract}
Modern production systems have numerous sensors that produce large amounts of data. This data can be exploited in many ways, from providing insight into the manufacturing process to facilitating automated decision making. These opportunities are still underexploited in the metal forming industry, due to the complexity of these processes. In this work, a probabilistic framework is proposed for simultaneous model improvement and state estimation in metal forming mass production. Recursive Bayesian estimation is used to simultaneously track the evolution of process state and to estimate the deviation between the physics-based model and the real process. A sheet bending mass production process is used to test the proposed framework. A metamodel of the process is built using proper orthogonal decomposition and radial basis function interpolation. The model is extended with a deviation model in order to account for the difference between model and real process. Particle filtering is used to track the state evolution and to estimate the deviation model parameters simultaneously. The approach is tested and analysed using a large number of simulations, based on pseudo-data obtained from a numerical sheet bending model.
\end{abstract}

Keywords Metal forming $\cdot$ State estimation $\cdot$ Hybrid modelling $\cdot$ Sheet bending $\cdot$ Mass production $\cdot$ Bayesian inference

\section{Introduction}

The metal forming industry is continuously challenged to develop processes with high throughput and precision, while minimizing costs and time-to-market. Process design in metal forming is knowledge-intensive and strongly dependent the designer's experience. Iterations between the design team and the press shop should be limited in order to minimize development time, but it is often inevitable to return to the drawing table when observations from the press shop do not match with the design team's predictions. The common response is to increase model complexity in order to reduce the discrepancy between model and reality. This is a labour intensive process that leads to only incremental progress.

Jos Havinga

jos.havinga@utwente.nl

1 Faculty of Engineering Technology, University of Twente, Enschede, Netherlands

2 Faculty of Electrical Engineering, Mathematics and Computer Science, University of Twente, Enschede, Netherlands
The Fourth Industrial Revolution (4IR) creates the opportunity for a paradigm shift in metal forming, by opening a direct connection between process design and the press shop. The connection is two-sided, as press shop observations can be used for model improvement, and models can be used for real-time interpretation of these observations. Data from the press shop can be employed to identify and characterize the deviations between model and reality, that originate either from deliberate simplification or ignorance of the actual physics [1]. Advanced process models which incorporate both data from physics-based models as well as production data can be used for process monitoring, predictive maintenance and real-time process control, potentially leading to unprecedented improvements in production accuracy and efficiency.

In this work, we propose a probabilistic framework for simultaneous model improvement and state estimation in metal forming mass production. The deviation between model and reality and the evolution of the process state will be estimated at the same time using recursive Bayesian estimation. The approach efficiently exploits data, as it serves two purposes at the same time. However, these objectives cannot be seen apart from each other, as state estimation requires accurate models and building accurate 
models is based upon processing large data streams from the manufacturing line.

The development of hybrid models that combine physicsbased models with real production data has the potential to tremendously impact general engineering [1] and the metal forming industry [2]. Although it is still difficult to find fully developed case studies of real-time data-driven modelling in metal forming, many researchers are already making significant steps towards this future prospect. The primary component is the development of data acquisition systems that collect production data on a product-to-product basis. Few examples are machine vision systems for ring rolling [3, 4], deepdrawing [5] and sheet rolling [6]; integrated sensors for local force measurement [7]; and toolintegrated distance measurement [8] and skid line detection [9] for stamping of car body parts. The development of such industrial metrology systems has led to the publication of several large metal forming datasets [8-11].

The key step to be taken is to determine a robust and accurate procedure for merging data from physics-based models with production data. Chinesta et al. [1] review several data aggregation techniques, for the development of so-called 'Hybrid Twins'. They focus on the combination with model order reduction, as real-time feedback for process control is a main application area for these models. They consider $\mathbf{x}(t)$ to be the system state (e.g., deformations and stresses) as a function of time $t$. The system behaviour can be described in rate form as $\dot{\mathbf{x}}(t, \boldsymbol{\theta})$, with constant parameters $\boldsymbol{\theta}$ (e.g., material and lubrication properties). ${ }^{1}$ The physics-based model is then given by $\dot{\mathbf{x}}(t, \boldsymbol{\theta})=\tilde{\mathbf{A}}(\mathbf{x}, t, \boldsymbol{\theta})$ and can be determined by offline computations. They define a deviation model (or bias model) $\Delta \mathbf{A}$ in additive form as function of system state and time, which has to be identified through data:

$\dot{\mathbf{x}}(t, \boldsymbol{\theta})=\tilde{\mathbf{A}}(\mathbf{x}, t, \boldsymbol{\theta})+\Delta \mathbf{A}(\mathbf{x}, t)$

Strano et al. [12] propose a similar idea and demonstrate an offline method for fusion of Finite Element (FE) and experimental data for air bending. They define a model in the measurement space instead of the state space. This model has a scalar output $\mathrm{z}$ (the angle after bending) and parametric input $\boldsymbol{\theta}$ (material and process parameters). An additive deviation model is then defined in the parameter space and determined through experiments:

$\mathrm{z}(\boldsymbol{\theta})=\tilde{\mathrm{A}}(\boldsymbol{\theta})+\Delta \mathrm{A}(\boldsymbol{\theta})$

\footnotetext{
${ }^{1}$ Some factors may be classified both as state or as parameters in different cases. Material properties can be considered as parameters in some cases, or as a system state when accounting for varying material properties during production.
}

These approaches for hybrid modelling are based on the assumption that the available datasets are complete; that is, each measurement contains a complete set of input and output values. However, such data may not always be available. In this work, we consider a metal forming production system where state $\mathbf{x}(t)$ varies over time. For instance, state variables may be material properties and/or lubrication properties. There may be unknown system parameters as well, such as machine compliance. During production, measurements $\mathbf{z}(\mathbf{x}, \boldsymbol{\theta})$ are taken (e.g., process forces). A measurement model $\tilde{\mathbf{z}}(\mathbf{x}, \boldsymbol{\theta})$ can be computed offline, but a measurement deviation model cannot be directly computed based on measurements from production, as the input values $(\mathbf{x}, \boldsymbol{\theta})$ that correspond to a specific measurement are unknown. As a first step to alleviate this problem, it can be assumed that the deviation model is independent of $\mathbf{x}$ and $\boldsymbol{\theta}$, and can be written as a mapping from modelled measurement to real measurement: $\mathbf{z}=\mathbf{g}\left(\tilde{\mathbf{z}}, \boldsymbol{\theta}_{d}\right)$, with a set of deviation parameters $\boldsymbol{\theta}_{d}$.

A single measurement $\mathbf{z}$ is insufficient to determine the deviation parameters $\boldsymbol{\theta}_{d}$, as different combinations of $\mathbf{x}, \boldsymbol{\theta}$ and $\boldsymbol{\theta}_{d}$ may result in the same measurement. Alternatively, one may exploit a series of measurements, such as the force measurements for multiple products within the mass production line. After each measurement, a set of the most probable deviation parameters and process states can be determined and propagated as input for the following measurement. By doing so, the problem is redefined in probabilistic terms. Probabilistic methods are hardly used in metal forming research (except for a few recent studies about material characterization [13, 14]), but widely used in many other areas (e.g., Kalman filtering for control systems). In this work, we present a framework for probabilistic state estimation in metal forming mass production. We aim at merging the predictive power of physics-based models and of real-time measurement data in order to maximize the quality of predictions during production. These predictions may be used to achieve improved performance of process monitoring and control systems.

The framework for real-time state estimation and model improvement in metal forming mass production is presented in Section "Recursive Bayesian state estimation". Modelling of a sheet bending case study is discussed in Section "Modelling". Results are presented in Section "Results", based on simulation runs with numerical pseudo-data. The effects of the quality of physics-based process models and of statistical assumptions on the algorithm performance are analysed in detail. A discussion follows in Section "Discussion". 


\section{Recursive Bayesian state estimation}

Industrial metal forming processes are subject to the influence of many factors that change over time, such as material, lubrication and process properties [15]. In this work, we refer to these properties as being the state $\mathbf{x}$ of the process. Variability of these properties has a strong effect on the final product properties [16]. Therefore, it is valuable to keep track of these properties during production. It is infeasible to directly measure the full process state during production, but valuable information about the state of the process can be obtained from indirect measurements such as process forces [11]. The value of the measurement data is determined by the sensitivity of the measurement to the state variables. Selection of appropriate measurement systems should therefore be based on the state variables of interest: force measurements may be sufficient to estimate the yield stress, whereas electromagnetic sensors may be required if estimation of the material microstructure is targeted.

In this work, we present a framework to infer a probabilistic estimate of the process state based on indirect measurements. The development of hybrid probabilistic models for state estimation will be discussed in Section "Process modelling". Subsequently, the framework for recursive Bayesian state estimation will be explained in Section "Recursive Bayesian estimation", and the numerical approach for solving the state estimation equations will be discussed in Section "Particle filtering".

\section{Process modelling}

A probabilistic framework requires probabilistic models, whereas metal forming models are typically deterministic. A partial probabilistic perspective in metal forming modelling is taken in the context of robust optimization, by optimizing the statistics of product properties given probabilistic assumptions about process variations [17]. A twofold extension of this probabilistic viewpoint is required for Bayesian state estimation: a probabilistic evolution mechanism for the process state as function of time (Section "State evolution model") and a probabilistic relation between process state and process measurement (Section "Measurement model") have to be defined. These models require estimates of process statistics and of model uncertainties. In Section "Deviation model", it is discussed how a deviation model can be used to aggregate data from physics-based models with production data.

\section{State evolution model}

In the context of mass production, the process state is discretized from product to product, with $\mathbf{x}_{i}$ being the state of the $i$-th product. The state evolution is the change in process state from product $i-1$ to the following product $i$. The evolution of the process state is assumed to be a Markov process, and is represented by the following discrete-time stochastic model:

$\mathbf{x}_{i}=\mathbf{f}\left(\mathbf{x}_{i-1}, \mathbf{v}_{i-1}\right)$

where $\mathbf{v}_{i-1}$ is a stochastic variable. Note that this assumption does not imply that the state of product $i$ is independent of its own deformation history, what would be incorrect when dealing with plasticity. The dependency of the state of product $i$ on the states of previous products can be extended by replacing (3) with a higher order Markov chain model.

Statistical characterization of material property variations is usually limited to obtaining mean values and standard deviations [18, e.g.,]. Some authors investigated the correlation between different material parameters [19, 20]. It is known that variations within material batches are smaller than variations between material batches [21], but little has been investigated about the autocorrelation of material properties in time. Therefore, we propose a simple model where each state variable is autocorrelated as:

$$
\begin{aligned}
& \mathrm{x}_{i}^{j}=\left(1-\rho^{j}\right) \mu^{j}+\rho^{j} \mathrm{x}_{i-1}^{j}+\mathrm{v}_{i-1}^{j} \\
& \mathrm{v}_{i-1}^{j} \sim \mathcal{N}\left(0,\left(1-\left(\rho^{j}\right)^{2}\right)\left(\sigma^{j}\right)^{2}\right)
\end{aligned}
$$

with $\mu^{j}, \sigma^{j}$ and $\rho^{j}$ being the mean value, standard deviation and correlation factor of the $j$-th state variable, respectively. The set of statistical parameters is $\boldsymbol{\theta}_{s}=\{\boldsymbol{\mu}, \boldsymbol{\sigma}, \boldsymbol{\rho}\}$. The value of $\rho^{j}$ can be $0 \leq \rho^{j} \leq 1$, with a larger value indicating stronger autocorrelation, i.e. slower changes in process state.

\section{Measurement model}

In Bayesian state estimation, the relation between process state $\mathbf{x}_{i}$ and process measurement $\mathbf{z}_{i}$ is given by a probabilistic measurement model:

$\mathbf{z}_{i}=\mathbf{h}\left(\mathbf{x}_{i}, \boldsymbol{\theta}, \mathbf{w}_{i}\right)$

with $\boldsymbol{\theta}$ being the constant model parameters and $\mathbf{w}_{i}$ being a stochastic variable that accounts for measurement uncertainty. Usually, measurement uncertainty is mostly related to sensor accuracy. In metal forming, however, model uncertainty is typically larger than measurement uncertainty. For example, process forces can be measured accurately, but the uncertainties in the models that relate these forces with the process state are much larger than the sensor inaccuracy itself.

The probabilistic approach requires a stochastic model for the measurement uncertainty. Quantification of model 
uncertainty is an open question in metal forming research. In this work, a simple estimate for the statistics of the measurement uncertainty $\mathbf{w}_{i}$ is made by determining the covariance of a numerical measurement dataset, which is built using Monte Carlo sampling and the prior statistics from the state variables and the constant parameters. The measurement uncertainty is assumed to be a fraction of this covariance.

\section{Deviation model}

Highly accurate models are required for interpreting small changes in measurement data in order to identify small changes in process state. Nowadays, detailed FE models are commonly used in metal forming. Although computer speed continuously increased in recent decades, model complexity and refinement have increased as well, and therefore, solution times for metal forming models remain high [22]. The problem of computational time can be circumvented through interpolation of a large set of offline model evaluations using metamodelling techniques [23], but the problem of limited accuracy remains due to modelling assumptions, as discussed in the introduction. Model accuracy may improve considerably by merging numerical data with data from process measurements. We propose to define a deviation model $\mathbf{z}=\mathbf{g}\left(\tilde{\mathbf{z}}, \boldsymbol{\theta}_{d}\right)$ that relates the modelled measurement $\tilde{\mathbf{z}}$ with the actual measurement $\mathbf{z}$ based on a set of deviation model parameters $\boldsymbol{\theta}_{d}$. The Bayesian state estimation procedure is suitable for hybrid modelling, as it allows for simultaneous estimation of unknown model parameters. The balance between model flexibility and estimation cost must be taken into account when defining a deviation model. A flexible deviation model is more likely to be able to map the actual deviation between model and reality, but will affect the efficiency of the state estimation procedure, as it will also require estimation of a larger set of parameters $\boldsymbol{\theta}_{d}$.

\section{Recursive Bayesian estimation}

In this work, the book by Ristic et al. [24] is used as reference on recursive Bayesian estimation and particle filtering (Section "Particle filtering"). It is assumed that the process state is continuous in space and discrete in time (each product is a sampling point in time). The measurements $\mathbf{Z}_{i} \triangleq\left\{\mathbf{z}_{k}\right\}_{k=1}^{i}$ are the observations from a hidden Markov model, with unobserved true states $\mathbf{x}$. Given the Markov process assumption, the probability density function (PDF) $p\left(\mathbf{x}_{i} \mid \mathbf{Z}_{i}\right)$ of the current state $\mathbf{x}_{i}$ given all the process observations $\mathbf{Z}_{i}$ can be obtained recursively in two steps. First, use the PDF $p\left(\mathbf{x}_{i-1} \mid \mathbf{Z}_{i-1}\right)$ of previous time point to obtain the predicted PDF of the current state given the past measurements as:

$p\left(\mathbf{x}_{i} \mid \mathbf{Z}_{i-1}\right)=\int p\left(\mathbf{x}_{i} \mid \mathbf{x}_{i-1}\right) p\left(\mathbf{x}_{i-1} \mid \mathbf{Z}_{i-1}\right) d \mathbf{x}_{i-1}$

where $p\left(\mathbf{x}_{i} \mid \mathbf{x}_{i-1}\right)$ is defined by the process model (3). Next, using the new measurement $\mathbf{z}_{i}$, the PDF can be updated (via Bayes theorem) to:

$p\left(\mathbf{x}_{i} \mid \mathbf{Z}_{i}\right) \propto p\left(\mathbf{z}_{i} \mid \mathbf{x}_{i}\right) p\left(\mathbf{x}_{i} \mid \mathbf{Z}_{i-1}\right)$

where $p\left(\mathbf{z}_{i} \mid \mathbf{x}_{i}\right)$ is defined by the measurement model (6). Equation 8 is given in proportional form, because it can be normalized using $\int p(\mathbf{x}) d \mathbf{x}=1$.

\section{Particle filtering}

In recursive Bayesian estimation, Eqs. 7 and 8 must be solved after each measurement. In case of linear systems and Gaussian noise, the Kalman Filter provides the optimal solution. In case of nonlinearity, suboptimal filters such as the Extended Kalman Filter or the Unscented Kalman Filter may be used to approximate the solution analytically. A different approach, which is especially useful for nonlinear models, is to use numerical methods to integrate the PDF's. Grid based methods integrate the full state space, but become extremely expensive for high-dimensional state spaces. Therefore, we use the particle filtering algorithm, which uses sequential Monte Carlo integration. The algorithm uses an importance sampling and a resampling step to concentrate the particles in the region of interest, neglecting the regions of low probability and reducing the curse of dimensionality. We use a standard particle filtering algorithm: the Sampling Importance Resampling (SIR) filter [25].

In the algorithm, the PDF's are approximated using $\mathrm{N}$ support points $\mathbf{x}_{i}^{k}$ and importance weights $w_{i}^{k}$ :

$p\left(\mathbf{x}_{i} \mid \mathbf{Z}_{i}\right) \approx \sum_{k=1}^{N} w_{i}^{k} \delta\left(\mathbf{x}_{i}-\mathbf{x}_{i}^{k}\right)$

The particle set $\left\{\mathbf{x}_{i}^{k}, w_{i}^{k}\right\}_{k=1}^{N}$ is determined using the particle set from the previous iteration $\left\{\mathbf{x}_{i-1}^{k}, w_{i-1}^{k}\right\}_{k=1}^{N}$, the new measurement $\mathbf{z}_{i}$, the process model (3) and the measurement model (6). The procedure is given in Algorithm 1. In SIR, the process model is used for importance sampling (drawing a set of particles at each iteration), but many other importance sampling strategies have been proposed in literature as well. After sampling, the particle weights $w_{i}^{k}$ are updated using the measurement 
model. Then, the weights are normalised, as $\int p(\mathbf{x}) d \mathbf{x}=1$. Finally, the particles are redistributed to the region of higher probability through resampling, eliminating the particles with negligible probability.

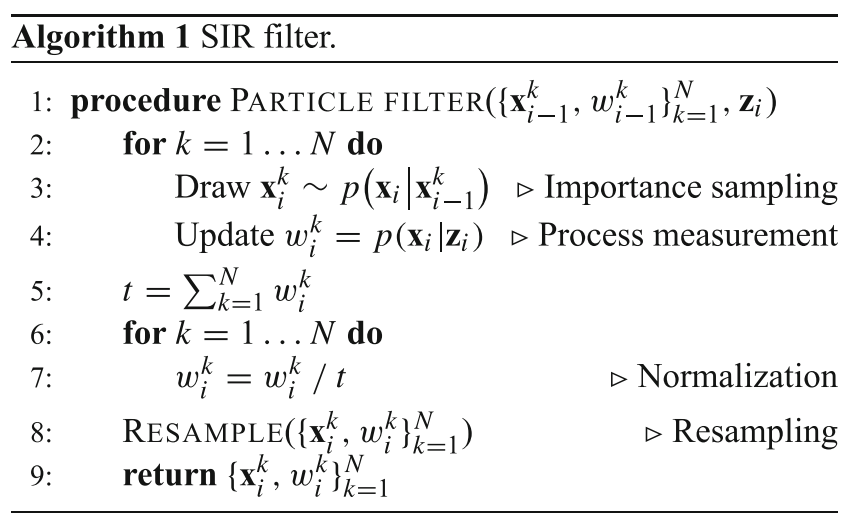

As discussed in the introduction, the state $\mathbf{x}$, constant parameters $\boldsymbol{\theta}$ and deviation model parameters $\boldsymbol{\theta}_{d}$ are estimated simultaneously. Towards that goal, the state space is augmented with the parameters. Thus, although different in nature, parameters $\boldsymbol{\theta}$ and $\boldsymbol{\theta}_{d}$ are treated as part of an extended state in the particle filtering algorithm. To avoid that the filter gets trapped in a wrong estimate of the parameters, a small variation (over time) is allowed in each parameter as follows [26]:

$\theta_{i}^{j}=\theta_{i-1}^{j}+u_{i}^{j}$

where the perturbations $u^{j}$ are independently sampled from $\mathcal{N}\left(0,\left(\alpha\left(\theta_{\max }^{j}-\theta_{\min }^{j}\right)\right)^{2}\right)$, with $\alpha$ typically in the order of 0.01 .

\section{Modelling}

The state estimation procedure is tested using data from a multistage mass production process. In this work, we focus on the flaps in the bottom of a demonstrator product (Fig. 2a), which are bent in two stages (Fig. 2b). The movement of contact areas during bending makes the process highly nonlinear. The bending force (Fig. 1) is measured during the first stage, and used to identify the process state. After the second bending stage, an image of one of the flaps is taken to determine the final angle (Fig. 2c). The used material is a $0.3 \mathrm{~mm}$ thick stainless steel sheet. Further information about the process can be found in Havinga et al. [11].

The FE model and metamodel of the process are presented in Sections "Finite element model" and "Metamodel", respectively. The deviation model that is used to identify the difference between the model and the real process is given in Section "Deviation model". The state

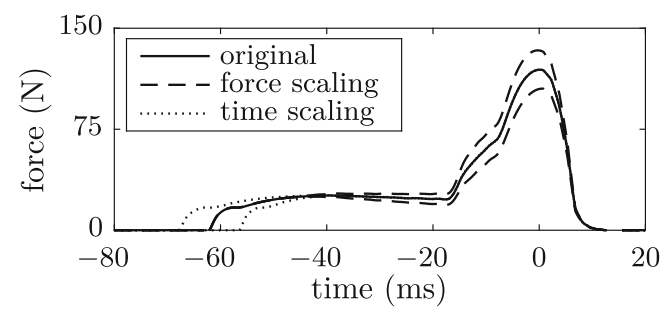

Fig. 1 Typical force during first bending stage, with effects of deviation parameters $\boldsymbol{\theta}_{d}$. The punch is at its lowest position at time $=0$

evolution model parameters are given in Section "State evolution model". Section "Simulation runs" provides an overview of all particle filtering simulations that have been performed.

\section{Finite element model}

The bending process is modelled in 2D using MSC.Marc. The tooling is modelled with 5370 elastic elements. A total of 3600 quadrilateral linear plane strain elements are used for the sheet (full integration with assumed strain option to avoid locking), with 15 elements through thickness. A Von Mises yield locus is used, with a tabulated hardening curve $\sigma_{0}(\varepsilon)$. Strain rate dependency is modelled using the Cowper-Symonds equation [27]:

$\sigma(\varepsilon, \dot{\varepsilon})=\left(1+\left(\frac{\dot{\varepsilon}}{\dot{\varepsilon}_{0}}\right)^{q}\right) \sigma_{0}(\varepsilon)$

The FE model has ten parameters that are shown in Fig. 3 and Table $1(\mathbf{x}$ and $\boldsymbol{\theta}$ ). These parameters are related to material, friction and tooling properties. A MATLAB procedure is used to automate the evaluation of large Designs of Experiments (DOE's). Model results are the force during first bending stage and the angles after both bending stages. The model evaluation time is approximately ten minutes.

\section{Metamodel}

The FE model solution time is too large for use in real-time applications. Therefore, a metamodel is built, which is a fast representation of the FE model. A large DOE is constructed, by combining a 2-level full factorial design $\left(2^{10}=1024\right.$ points) with a latin hypercube design (6000 points). A small number of simulations crashed, and the remaining 6953 simulation results are interpolated using radial basis function interpolation [17]. Separate metamodels are built for the angles after both bending stages. Proper Orthogonal Decomposition (POD) is used to reduce the result space of the force curves to a set of 40 modes, that are interpolated independently [23]. The final interpolation model has an evaluation time of approximately $10 \mathrm{~ms}$. 


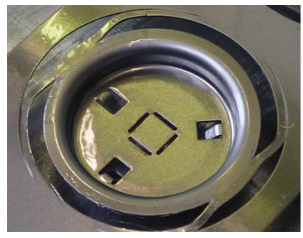

(a) product

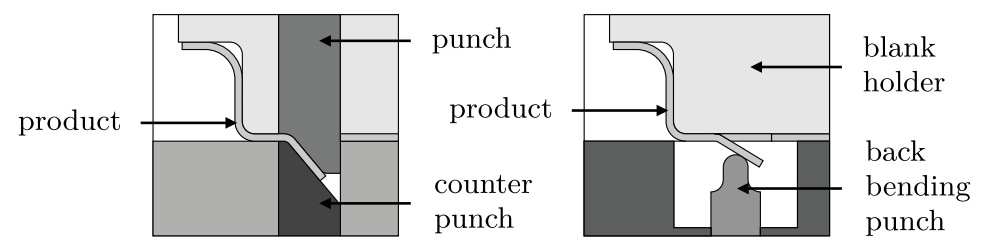

(b) First and second bending stage

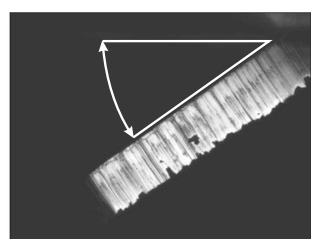

(c) Picture of flap from inline camera

Fig. 2 Demonstrator process [11]

The chosen offline metamodeling procedure is suitable for the studied case, as only product-to-product variation is considered, and no evolution of the state (e.g. deformation or plastic strain) is being tracked during forming of a single product. Furthermore, the dimensionality of the problem is moderate. Therefore, it is feasible to characterize the process behavior throughout the full state space using offline computations. In other cases, it may be required to solve constitutive equations almost in real-time. Several methods are being developed that allow to solve nonlinear state evolution equations simultaneously with identification of unknown model parameters. These methods are based on model order reduction techniques such as Proper Generalized Decomposition (PGD), and are specifically suitable for high-dimensional problems [28, 29].

\section{Deviation model}

A key objective of this work is to construct a 'Hybrid Twin', by mapping the modelled force measurement $\tilde{\mathbf{z}}$ to the actual force measurement $\mathbf{z}$, in order to reduce the gap between the process metamodel and reality. The mapping $\mathbf{z}=\mathbf{g}\left(\tilde{\mathbf{z}}, \boldsymbol{\theta}_{d}\right)$ is defined by a set of deviation parameters $\boldsymbol{\theta}_{d}$, and is assumed to be independent of the input space $\mathbf{x}$. A mapping function $\mathbf{g}$ must be chosen such that it is sufficiently flexible to capture the difference between model and reality, but

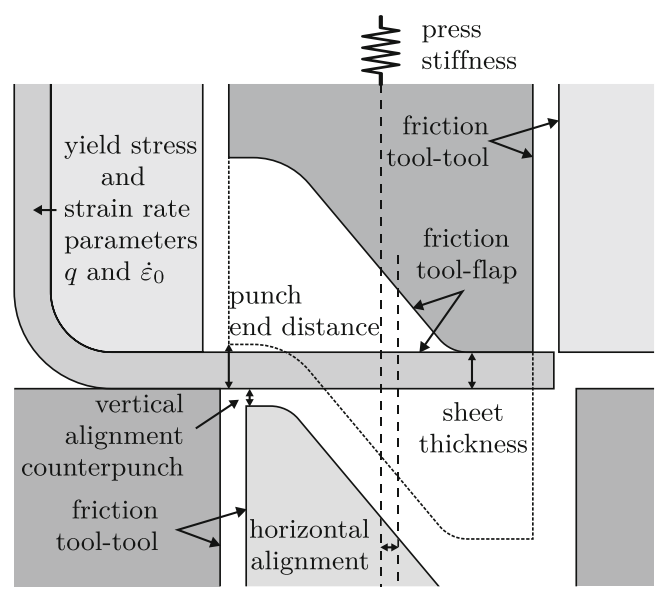

Fig. 3 FE model parameters without too many parameters to be identified. In this work, $\boldsymbol{\theta}_{d}$ is a set of scaling factors for both force and punch speed. The scaling parameters are defined at four evenly spaced points between the minimum $(-80 \mathrm{~ms})$ and maximum $(20 \mathrm{~ms})$ simulation time, with linear interpolation in between in order to ensure continuity of the force curves. The effect of these scaling parameters is illustrated in Fig. 1: speed scaling stretches or compresses the force curve in time, and force scaling increases or decreases the magnitude of the force. In that way, a consistently incorrect prediction in a particular part of the force curve can be corrected by the deviation model.

\section{State evolution model}

The state evolution is modelled using Eq. 4. The values for standard deviations $\sigma$ and correlation parameters $\rho$ are listed in Table 1. Different datasets have been used for the simulation runs (see Section "Simulation runs"), and different values of $\boldsymbol{\mu}$ have been used in each dataset. These values for $\boldsymbol{\mu}$ are randomly sampled from a uniform distribution between $\boldsymbol{\mu}_{\min }$ and $\boldsymbol{\mu}_{\max }$ (Table 1).

\section{Simulation runs}

The state estimation procedure is tested using simulation runs with pseudo-data. An advantage of using pseudo-data, is that the estimation errors can be determined exactly, as the 'real' state $\mathbf{x}$ and parameters $\boldsymbol{\theta}$ are fully known. Six different series of particle filtering simulations are performed, in order to obtain understanding about the applicability of particle filtering for state estimation in metal forming mass production. These simulation series are chosen such that the effects of different error sources (i.e. measurement model accuracy and process model accuracy) can be studied separately.

The six simulation series are performed with different measurement datasets and particle filter settings (see Fig. 4). Five different realizations of the input data $\left(\left\{\mathbf{x}_{i}\right\}_{i=1}^{1000}, \boldsymbol{\theta}, \boldsymbol{\theta}_{d}\right)$ are used for all simulation series. The 5 realizations of the state evolution $\left\{\mathbf{x}_{i}\right\}_{i=1}^{1000}$ are generated using Eq. 4, by considering 5 different sets of statistical parameter $\boldsymbol{\theta}_{S^{-}}$ values. All parameter values $\left(\boldsymbol{\theta}, \boldsymbol{\theta}_{d}, \boldsymbol{\theta}_{s}\right)$ are drawn from 
Table 1 Model parameters

\begin{tabular}{|c|c|c|c|c|c|c|c|}
\hline & \multirow[b]{2}{*}{ Unit } & \multirow[b]{2}{*}{ Min } & \multirow[b]{2}{*}{ Max } & \multicolumn{4}{|l|}{$\boldsymbol{\theta}_{s}$} \\
\hline & & & & $\mu_{\min }$ & $\mu_{\max }$ & $\sigma$ & $\rho$ \\
\hline \multicolumn{8}{|l|}{$\mathbf{x}$} \\
\hline yield stress & Mpa & 275 & 336 & 291 & 321 & 6 & 0.8 \\
\hline mat. param. $\log \left(\dot{\epsilon}_{0}\right)$ & $\log (1 / s) 1.5$ & 2.5 & 2 & 2.4 & 0.05 & 0.8 & \\
\hline material param. $q$ & - & 0.4 & 1.0 & 0.7 & 0.9 & 0.05 & 0.8 \\
\hline sheet thickness & $\mu \mathrm{m}$ & 295 & 305 & 298 & 302 & 1 & 0.7 \\
\hline fric. coeff. tool-tool & - & 0.1 & 0.5 & 0.2 & 0.3 & 0.05 & 0.95 \\
\hline fric. coeff. tool-flap & - & 0.01 & 0.3 & 0.1 & 0.2 & 0.02 & 0.95 \\
\hline punch end distance & $\mu \mathrm{m}$ & 310 & 410 & 340 & 380 & 10 & 0.9 \\
\hline \multicolumn{8}{|l|}{$\theta$} \\
\hline horizontal alignment & $\mu \mathrm{m}$ & -5 & 20 & & & & \\
\hline vertical alignment & $\mu \mathrm{m}$ & -20 & 0 & & & & \\
\hline press stiffness & $\mathrm{kN} / \mathrm{mm}$ & 5 & 40 & & & & \\
\hline \multicolumn{8}{|l|}{$\boldsymbol{\theta}_{d}$} \\
\hline force scaling & $\%$ & -20 & 20 & & & & \\
\hline speed scaling & $\%$ & -20 & 20 & & & & \\
\hline
\end{tabular}

uniform distributions within the bounds given in Table 1 (with $\boldsymbol{\mu}_{\min } \leq \boldsymbol{\mu} \leq \boldsymbol{\mu}_{\max }$ ). For each combination of simulation series and dataset realization, the particle filtering simulation is repeated 5 times, as the algorithm involves random sampling, leading to different results for each simulation with exactly the same data and settings. Hence, a total of 6 simulation series $\times 5$ dataset realizations $\times 5$ repetitions $=150$ particle filtering simulations are performed.

The differences between the 6 simulation series are listed in Fig. 4. These series use different measurement datasets or particle filter settings. The measurement datasets are either directly obtained from the metamodel $\left(\tilde{\mathbf{z}}^{\mathrm{MM}}\right)$ or from the FE model $\left(\tilde{\mathbf{z}}^{\mathrm{FE}}\right)$, or they are altered using the deviation model $\left(\mathbf{g}\left(\tilde{\mathbf{z}}^{\mathrm{MM}}, \boldsymbol{\theta}_{d}\right)\right.$ or $\left.\mathbf{g}\left(\tilde{\mathbf{z}}^{\mathrm{FE}}, \boldsymbol{\theta}_{d}\right)\right)$. Similarly, the metamodel that is used by the particle filter may or may not include the deviation model $\left(\mathbf{g}\left(\operatorname{MM}(\mathbf{x}, \boldsymbol{\theta}), \boldsymbol{\theta}_{d}\right)\right.$ or $\operatorname{MM}(\mathbf{x}, \boldsymbol{\theta})$, respectively). The statistical parameters $\boldsymbol{\theta}_{s}$ are exactly known by the particle filter, except for series 4 and 6 , where the value of $\boldsymbol{\mu}$ is assumed to be $\left(\boldsymbol{\mu}_{\min }+\boldsymbol{\mu}_{\max }\right) / 2{ }^{2}$

The assumption for the measurement uncertainty $\mathbf{w}(6)$ is computed offline, based on a measurement dataset $\mathbf{Z}_{M C}$ of $10^{4}$ products obtained by Monte Carlo sampling of the combined state $\mathbf{x}$ and parameter $\boldsymbol{\theta}$ space, using the prior statistics, with $\rho=\mathbf{0}$. In case of the simulation series with unknown statistics, $\boldsymbol{\mu}$ is again assumed to be $\left(\boldsymbol{\mu}_{\min }+\right.$ $\left.\boldsymbol{\mu}_{\max }\right) / 2$. The $10^{4}$ points are evaluated using the metamodel. The covariance of $\mathbf{Z}_{M C}$ is $\mathbf{K}_{M C}$. The covariance of the measurement uncertainty $\mathbf{w}$ is assumed to be $\left(\frac{1}{2}\right)^{2} \mathbf{K}_{M C}$

\footnotetext{
The value $(\max -\min ) / \sqrt{6}$ is the expected value of the RMSE for a random estimator with uniform PDF.
}

(i.e. the standard deviation of measurement uncertainty $\mathbf{w}$ is assumed to be half of the standard deviation of $\mathbf{K}_{M C}$ ). As the trial dataset covariance $\mathbf{K}_{M C}$ is determined in the result space without model deviation, the reverse deviation is applied to the measurement, before comparing the measurement with the model prediction. The full measurement model becomes:

$\mathbf{g}^{-1}\left(\mathbf{z}, \boldsymbol{\theta}_{d}\right) \sim \mathcal{N}\left(\tilde{\mathbf{z}}(\mathbf{x}, \boldsymbol{\theta}),\left(\frac{1}{2}\right)^{2} \mathbf{K}_{M C}\right)$

The number of particles $N$ in the particle filter is set to 500. The angles after first and second bending stage are not used by the particle filter to estimate the process state $\mathbf{x}$ and parameters $\boldsymbol{\theta}$ and $\boldsymbol{\theta}_{d}$. Hence, the process state is estimated based on only process forces. However, the estimates of $\mathbf{x}$ and $\boldsymbol{\theta}$ are used to predict the angles as well, which are then compared with the real angles in the pseudo-dataset.

\section{Results}

The estimated state/parameter value $\mathbb{E}\left[\mathbf{x}_{i}\right]$ and the standard deviation $\mathbf{s}_{i}$ for the $i$-th product are determined with:

$$
\begin{aligned}
& \mathbb{E}\left[\mathbf{x}_{i}\right]=\sum_{k=1}^{N} w_{i}^{k} \mathbf{x}_{i}^{k} \\
& \mathbf{s}_{i}=\sqrt{\sum_{k=1}^{N} w_{i}^{k}\left(\mathbf{x}_{i}^{k}-\mathbb{E}\left[\mathbf{x}_{i}\right]\right)^{2}}
\end{aligned}
$$

The Root-Mean-Square Error (RMSE) between the actual and the predicted state/parameter values are calculated for all simulation series and normalized for comparison 


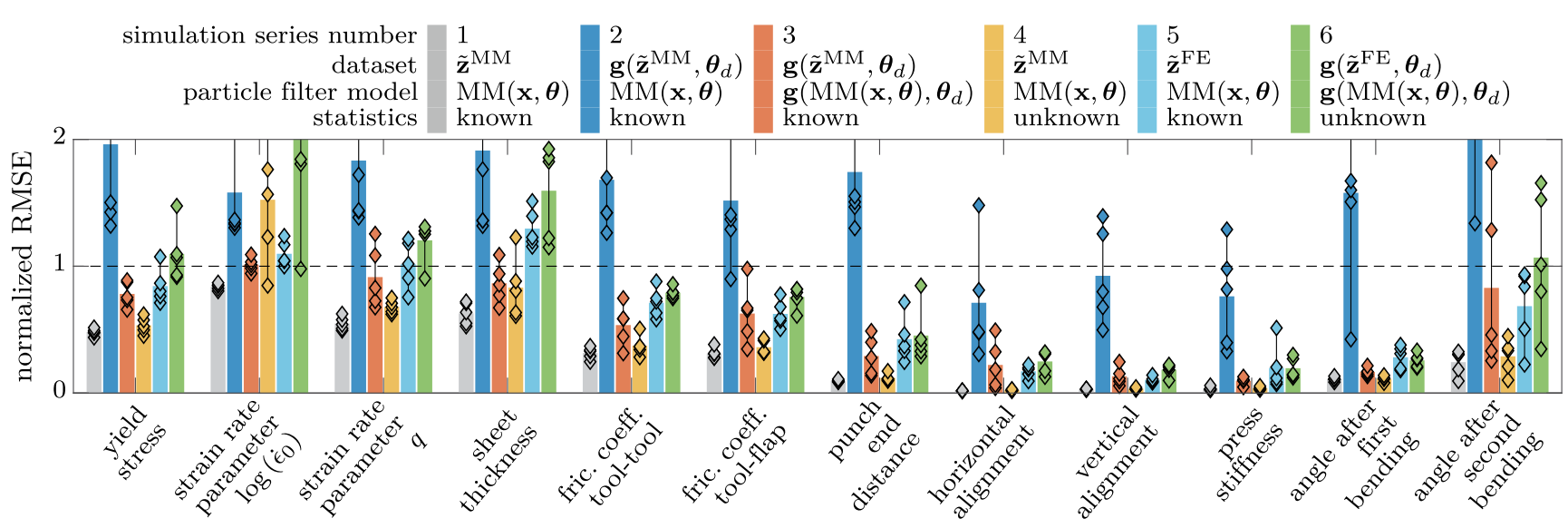

Fig. 4 Average RMSE per state variable and parameter, calculated over the last 500 products of each simulation and averaged over all datasets. The RMSE values per dataset (averaged over five repetitions) are indicated with diamonds. The RMSE values are normalized by $\sigma$ (Table 1$)$ for the state variables, by $(\max -\min ) / \sqrt{6}$ for the constant

purposes (normalization is explained in the caption of Fig. 4). The reference is series 1 , where the metamodel without deviation model is used both to generate the data as well as for estimation. In other words, the particle filter has a 'perfect' model of the real process. The RMSE values for series 1 give an indication about the observability of the state $\mathbf{x}$ and parameters $\boldsymbol{\theta}$ based on force measurements. The average RMSE values for all series are shown in Fig. 4. It can be seen for series 1 that the estimation accuracy differs per parameter: punch end distance and the constant parameters can be estimated with high accuracy, whereas the material parameters and thickness are harder to estimate. This can also be seen in Fig. 5, where the actual values and estimates are shown for the last 200 products for one of the simulations from series 1 . The state $\mathbf{x}$ and parameter $\boldsymbol{\theta}$ estimates are used to predict the angle after first and second bending, which have an average normalized RMSE of 0.11 and 0.24 respectively. It can be concluded that the force measurements contain sufficient information to obtain a good estimate of the state $\mathbf{x}$, parameters $\boldsymbol{\theta}$ and angles. It must be noted that these results will be affected by the chosen statistical parameters $\boldsymbol{\theta}_{s}$, as small variations in process state are harder to identify than large variations.

The worst case reference is series 2, which is performed using dataset $\mathbf{g}\left(\tilde{\mathbf{z}}^{\mathrm{MM}}, \boldsymbol{\theta}_{d}\right)$ (i.e., with simulated deviation between 'real' data and process model), but estimated using a model that does not account for the deviation. As the model used by the particle filter is incorrect, it is unable to find a correct estimate for any of the state variables or parameters (Fig. 4). However, this does not mean that the model is entirely wrong, as the used dataset is obtained from the same metamodel (but transformed with the deviation model afterwards). The problem for the state estimation procedure is that the measured data does not map correctly parameters $^{2}$, and by the standard deviation of the real angles for the angles. A RMSE larger than 1 indicates that the prediction error is larger than the variation in the data (for the state $\mathbf{x}$ and the angles) or that the estimator performs worse than a random estimator with uniform PDF (for the constant parameters $\boldsymbol{\theta}$ )

to the model. This is similar to the case where real data from the press shop is used for state estimation: the model may be 'correct' in the sense that it correctly predicts the major
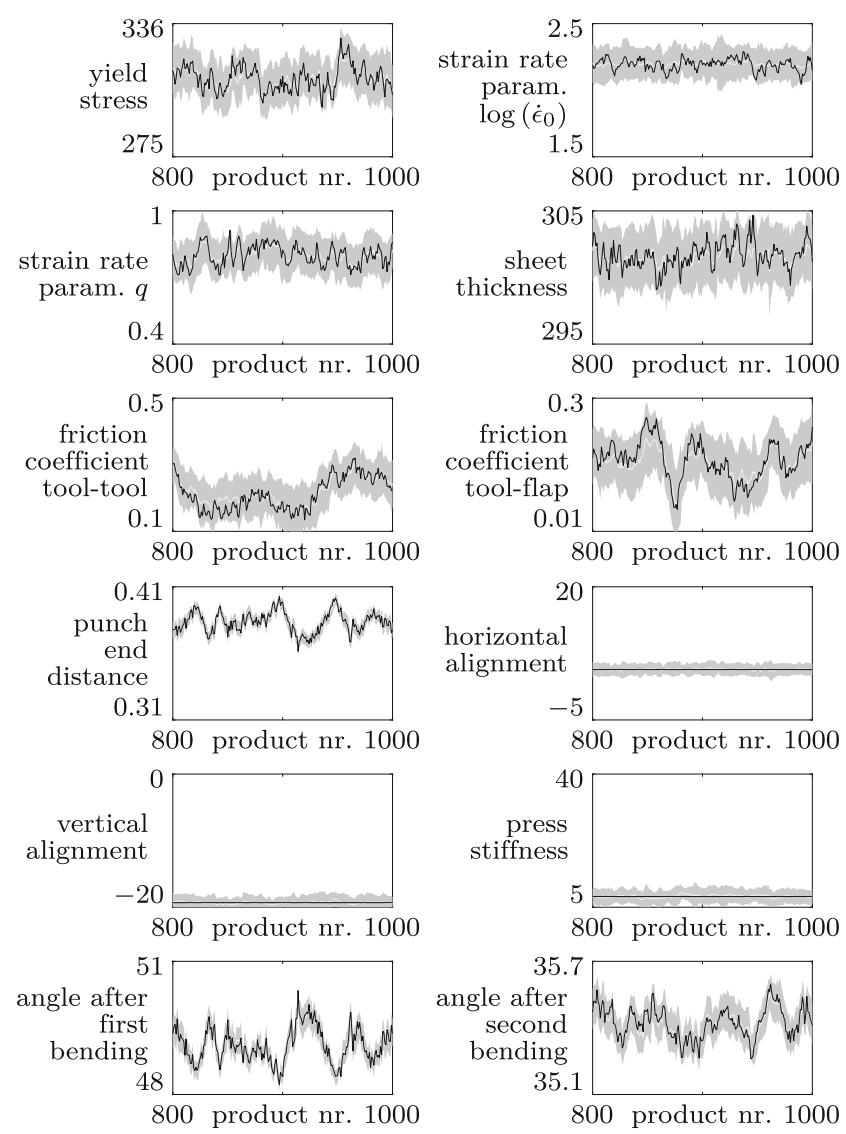

Fig. 5 Predicted (grey) and actual (black) state/parameter values for a simulation from simulation series 1, i.e. with 'perfect' particle filter model. The prediction bands are plotted with $\mathbb{E}\left[\mathbf{x}_{i}\right] \pm 3 \mathbf{s}_{i}(99.7 \%$ confidence interval) 
part of the underlying physics, but it is not usable for state estimation as long as the gap between model and reality leads to incorrect mapping of model data to the real data.

Through this work, we hypothesize that the difference between the process model and reality can be estimated simultaneously with the process state through probabilistic methods. This is done in series 3, again using dataset $\mathbf{g}\left(\tilde{\mathbf{z}}^{\mathrm{MM}}, \boldsymbol{\theta}_{d}\right)$, but now with simultaneous estimation of the eight deviation parameters $\boldsymbol{\theta}_{d}$. A reasonable estimate of the process state and the angles after bending is obtained in this simulation series. This is indicative for the potential of simultaneous model improvement and state estimation. It must be, however, noted that the actual deviation between model and real data for series 3 is known to be present in the deviation parameter space (as the data from series 3 is generated using exactly the same deviation model). This is not known a priori when working with real process data. As with any fitting problem, it is the challenge to choose a model that is sufficiently flexible to describe the behaviour of the data, without being too flexible, making it expensive to fit and prone to overfitting.

The accuracy of the statistical models also has a significant effect on estimation performance. The only difference between series 4 and reference series 1 is that the particle filter in series 4 uses incorrect values for $\boldsymbol{\mu}$ (4). The estimate is therefore biased towards the incorrect mean, leading larger errors. Obviously, a larger deviation between modelled statistics and actual statistics will increase the prediction error. As discussed in Section "State evolution model", little is known about statistics in metal forming, making it is difficult to calibrate statistical models. A possible solution is to treat the statistical parameters as hyperparameters to be identified during the estimation procedure as well [30].

The final two simulation series are performed with data that is obtained directly from the FE model, instead of from the metamodel. Series 5 is performed without deviation in the data, isolating the effect of the difference between FE model and metamodel. The prediction accuracy is lower than for the reference series, but it is still possible to obtain a reasonable estimate of state $\mathbf{x}$, parameters $\boldsymbol{\theta}$ and angles. The final series (nr. 6) gives the most complete comparison with reality: the model (MM) and the real process (FE) are different, a deviation model is used to increase the gap between model and reality, and statistical parameters are partly unknown. In Fig. 4 it can be seen that the prediction error is much larger than for the perfect reference series, but still sufficiently accurate to be able to identify part of the variations in the process. Fig. 6 gives a detailed view of the estimation for the last 200 products from one of the simulations from series 6 . The prediction errors are larger than in the reference series (Fig. 5). However, it can be seen that trends in the state evolution are still detected, even though the estimated values are incorrect. Such estimates
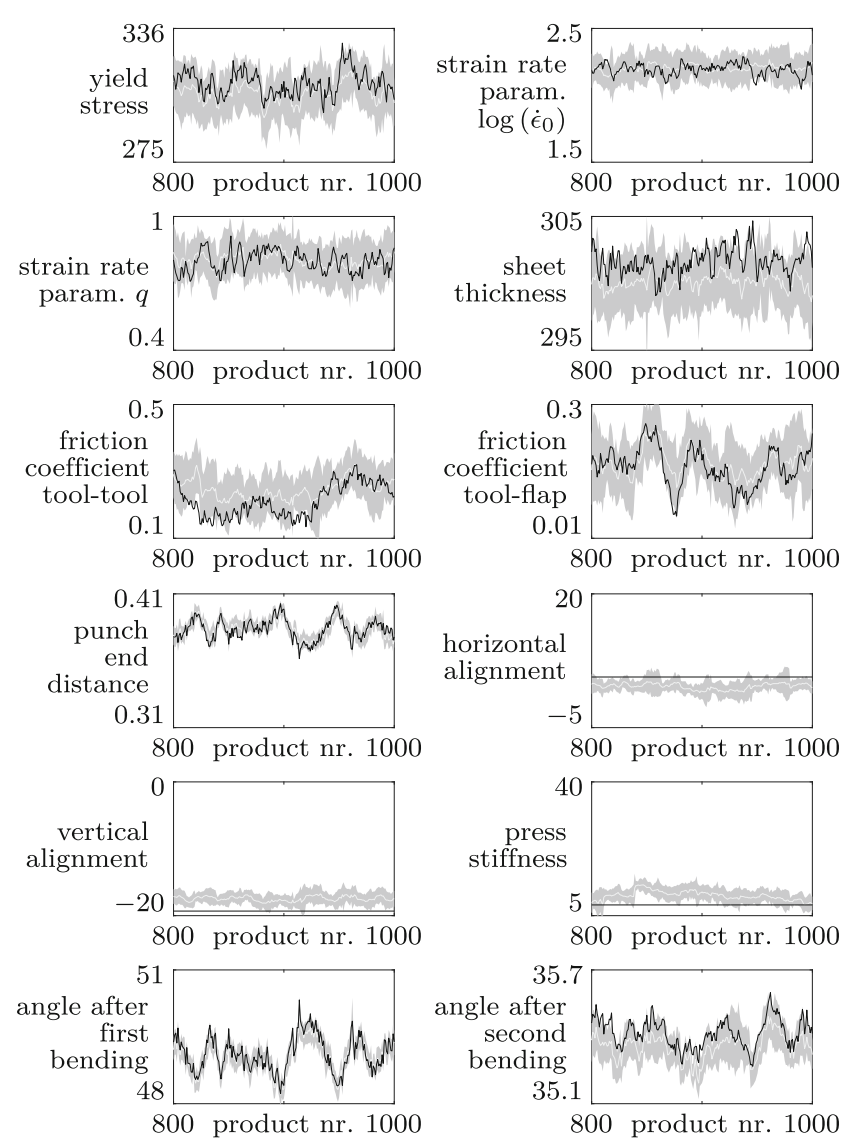

Fig. 6 Predicted (grey) and actual (black) state/parameter values for a simulation from series 6 , i.e. with $\mathbf{g}\left(\operatorname{MM}(\mathbf{x}, \boldsymbol{\theta}), \boldsymbol{\theta}_{d}\right)$ model being used to estimate $\mathbf{g}\left(\tilde{\mathbf{z}}^{\mathrm{FE}}, \boldsymbol{\theta}_{d}\right)$ data. The prediction bands are plotted with $\mathbb{E}\left[\mathbf{x}_{i}\right] \pm 3 \mathbf{s}_{i}(99.7 \%$ confidence interval)

of trends in the process state are still highly valuable when used for process monitoring and control. In conclusion, the proposed approach shows clear potential for added value in industrial metal forming mass production, but it still has many factors that need to be improved before reaching a sufficient level of maturity for industrial applicability.

\section{Discussion}

An approach for simultaneous model improvement and state estimation for metal forming mass production is proposed in this work. Recursive Bayesian methods are widely used in many application areas, but not yet in metal forming. The 4IR opens the opportunity for the development of systems that are dependent on real-time acquisition and processing of manufacturing data. We foresee that metal forming research will develop towards a hybrid approach, where knowledge is obtained both from detailed physicsbased models as well as from large manufacturing data streams. The proposed approach is indicative of this route, 
by combining traditional modelling with learning from process data.

The recursive Bayesian estimation procedure is tested using pseudo-data from a very nonlinear bending mass production process. The results illustrate the potential of the approach. However, several challenges for further research are faced, being:

Deviation model Hybrid models that merge data from physics-based models with real data will be a key component of future smart manufacturing systems. The development of deviation models for very nonlinear metal forming processes is extremely challenging, as they must capture what physics-based models fail to capture. This research will be fuelled by the increasing interest from metal forming researchers in machine learning methods.

Measurement uncertainty In this work, a simple measurement uncertainty model is used, even while performing the analysis with pseudo-data. Understanding about the nature of model uncertainty and measurement accuracy in metal forming is an open research question, that becomes highly relevant when using probabilistic methods.

Process evolution The process evolution model is a key component of the state estimation procedure. However, little is known about variability of metal forming processes, both regarding appropriate models as well as statistics thereof. Increasingly available data from manufacturing lines will enable the development of better process evolution models.

Algorithm development An established particle filter algorithm is used in this work, without optimization for the specific application. The applicability of different algorithms must be investigated in order to develop fast, robust and accurate algorithms that are tailor-made for metal forming applications.

Learning from data and interpreting data in real-time are two key promises of the 4IR. We propose a probabilistic framework that connects these objectives and enables the inclusion of knowledge from physics-based models into the manufacturing line. Several challenges still have to be addressed, in order to move towards the use of truly smart models in modern factories.

\section{Compliance with Ethical Standards}

Conflict of interests The authors declare that they have no conflict of interest.

Open Access This article is distributed under the terms of the Creative Commons Attribution 4.0 International License (http:// creativecommons.org/licenses/by/4.0/), which permits unrestricted use, distribution, and reproduction in any medium, provided you give appropriate credit to the original author(s) and the source, provide a link to the Creative Commons license, and indicate if changes were made.

\section{References}

1. Chinesta F, Cueto E, Abisset-Chavanne E, Duval JL, Khaldi FE (2018) Virtual, digital and hybrid twins: a new paradigm in data-based engineering and engineered data. Archives of Computational Methods in Engineering

2. Post J, Groen M, Klaseboer G (2017) Physical model based digital twins in manufacturing processes. In: Model based control for smart forming processes, proceedings of 10th forming technology forum. University of Twente, pp 87-92

3. Arthington MR, Cleaver C, Allwood J, Duncan S (2015) Measurement and control of variable geometry during ring rolling. In: 2015 IEEE conference on control applications (CCA). IEEE

4. Husmann S, Kuhlenkötter B (2017) Investigation for an automated avoidance of ring climbing in radial-axial ring rolling. Procedia Eng 207:1242-1247

5. Fischer P, Heingärtner J, Renkci Y, Hora P (2018) Experiences with inline feedback control and data acquisition in deep drawing. Procedia Manuf 15:949-954

6. Carruthers-Watt BN, Xue Y, Morris AJ A vision based system for strip tracking measurement in the finishing train of a hot strip mill. In: 2010 IEEE international conference on mechatronics and automation. IEEE, vol 2010

7. Groche P, Hohmann J, Übelacker D (2019) Overview and comparison of different sensor positions and measuring methods for the process force measurement in stamping operations. Measurement 135:122-130

8. Maier S, Liebig A, Kautz T, Volk W (2017) Tool-integrated spring back measuring system for automotive press shops. Prod Eng 11(3):307-313

9. Maier S, Schmerbeck T, Liebig A, Kautz T, Volk W (2017) Potentials for the use of tool-integrated in-line data acquisition systems in press shops. J Phys Con Series 896:012033

10. Purr S, Wendt A, Meinhardt J, Moelzl K, Werner A, Hagenah $H$, Merklein M (2016) Data-driven inline optimization of the manufacturing process of car body parts. In: Proceedings of the IDDRG 2016 conference, pp 200-209

11. Havinga J, van den Boogaard T, Dallinger F, Hora P (2018) Feedforward control of sheet bending based on force measurements. $\mathrm{J}$ Manuf Process 31:260-272

12. Strano M, Iorio L, Semeraro Q, Sofia R (2017) Fusion metamodeling of the bend deduction in air bending. In: AIP Conference proceedings, Author(s), vol 1896, p 100003

13. Asaadi E, Heyns PS (2017) A computational framework for bayesian inference in plasticity models characterisation. Comput Methods Appl Mech Eng 321:455-481

14. Cornaggia A, Cocchetti G, Maier G, Buljak V (2018) Inverse structural analyses on small punch tests, with model reduction and stochastic approach. In: 2018 IEEE International conference on environment and electrical engineering and 2018 IEEE industrial and commercial power systems Europe (EEEIC / I\&CPS Europe). IEEE

15. Col A (2003) Investigation on press forming scatter origin. In: Proceedings of the sixth international ESAFORM conference on material forming, pp 183-186

16. Hazra S, Williams D, Roy R, Aylmore R, Smith A (2011) Effect of material and process variability on the formability of aluminium alloys. J Mater Process Technol 211(9):1516-1526 
17. Havinga J, van den Boogaard AH, Klaseboer G (2016) Sequential improvement for robust optimization using an uncertainty measure for radial basis functions. Struct Multidiscip Optim 55(4):13451363

18. Hess PE, Bruchman D, Assakkaf IA, Ayyub BM (2002) Uncertainties in material and geometric strength and load variables. Nav Eng J 114(2):139-166

19. Aspenberg D, Larsson R, Nilsson L (2012) An evaluation of the statistics of steel material model parameters. J Mater Process Technol 212(6):1288-1297

20. Wiebenga J, Atzema E, An Y, Vegter H, van den Boogaard A (2014) Effect of material scatter on the plastic behavior and stretchability in sheet metal forming. J Mater Process Technol 214(2):238-252

21. Hora P, Heingärtner J, Manopulo N, Tong L (2011) Zero failure production methods based on a process integrated virtual control. In: AIP Conference proceedings, AIP, vol 1383, pp $35-47$

22. Allwood J, Duncan S, Cao J, Groche P, Hirt G, Kinsey B, Kuboki T, Liewald M, Sterzing A, Tekkaya A (2016) Closed-loop control of product properties in metal forming. CIRP Ann 65(2):573596

23. Buljak V, Maier G (2011) Proper orthogonal decomposition and radial basis functions in material characterization based on instrumented indentation. Eng Struct 33(2):492-501
24. Ristic B, Arulampalam S, Gordon N (2004) Beyond the Kalman Filter, 1st edn. Artech House Publishers

25. Gordon N, Salmond D, Smith A (1993) Novel approach to nonlinear/non-gaussian bayesian state estimation. IEE Proceedings F Radar and Signal Processing 140(2):107

26. Liu J, West M (2001) Combined parameter and state estimation in simulation-based filtering. In: Sequential Monte Carlo methods in practice. Springer, New York, pp 197-223

27. Cowper CR, Symonds PS (1957) Strain-hardening and strain-rate effects in the impact loading of cantilever beams. Tech. Rep 28, Division of applied mathematics, Brown University

28. Gonzàlez D, Badías A, Alfaro I, Chinesta F, Cueto E (2017) Model order reduction for real-time data assimilation through extended Kalman filters. Comput Methods Appl Mech Eng 326:679-693

29. Marchand B, Chamoin L, Rey C (2019) Parameter identification and model updating in the context of nonlinear mechanical behaviors using a unified formulation of the modified constitutive relation error concept. Comput Methods Appl Mech Eng 345:1094-1113

30. Storvik G (2002) Particle filters for state-space models with the presence of unknown static parameters. IEEE Trans Signal Process 50(2):281-289

Publisher's note Springer Nature remains neutral with regard to jurisdictional claims in published maps and institutional affiliations. 